\title{
\begin{tabular}{l|l} 
Mitraries & DSpace@MIT
\end{tabular}
}

\author{
MIT Open Access Articles
}

\section{A simple and accurate discontinuous Galerkin scheme for modeling scalar-wave propagation in media with curved interfaces}

The MIT Faculty has made this article openly available. Please share how this access benefits you. Your story matters.

Citation: Zhang, Xiangxiong, and Sirui Tan. "A Simple and Accurate Discontinuous Galerkin Scheme for Modeling Scalar-Wave Propagation in Media with Curved Interfaces." GEOPHYSICS 80, no. 2 (January 14, 2015): T83-T89. (c) 2015 Society of Exploration Geophysicists

As Published: http://dx.doi.org/10.1190/geo2014-0164.1

Publisher: Society of Exploration Geophysicists

Persistent URL: http://hdl.handle.net/1721.1/97123

Version: Final published version: final published article, as it appeared in a journal, conference proceedings, or other formally published context

Terms of Use: Article is made available in accordance with the publisher's policy and may be subject to US copyright law. Please refer to the publisher's site for terms of use. 


\title{
A simple and accurate discontinuous Galerkin scheme for modeling scalar-wave propagation in media with curved interfaces
}

\author{
Xiangxiong Zhang ${ }^{1}$ and Sirui $\operatorname{Tan}^{2}$
}

\begin{abstract}
Conventional high-order discontinuous Galerkin (DG) schemes suffer from interface errors caused by the misalignment between straight-sided elements and curved material interfaces. We have developed a novel DG scheme to reduce those errors. Our new scheme uses the correct normal vectors to the curved interfaces, whereas the conventional scheme uses the normal vectors to the element edge. We modify the numerical fluxes to account for the curved interface. Our numerical modeling examples demonstrate that our new discontinuous Galerkin scheme gives errors with much smaller magnitudes compared with the conventional scheme, although both schemes have second-order convergence. Moreover, our method significantly suppresses the spurious diffractions seen in the results obtained using the conventional scheme. The computational cost of our scheme is similar to that of the conventional scheme. The new DG scheme we developed is, thus, particularly useful for large-scale scalarwave modeling involving complex subsurface structures.
\end{abstract}

\section{INTRODUCTION}

Discontinuous Galerkin (DG) finite-element methods have been developed for a wide range of problems since their inception in the 1970s (e.g., Reed and Hill, 1973; Cockburn and Shu, 1989; Hesthaven and Warburton, 2007). In contrast to classical continuous finite-element methods, the solution of DG methods can be discontinuous across element interfaces. The weak coupling between elements makes the mass matrix local to the cell, and thus, DG schemes are compact because only communication with immediate neighbors is needed. DG methods are attractive choices for handling complicated geometries and allowing hanging nodes in the mesh. DG methods have gained popularity for seismic modeling since the work of Käser and Dumbser (2006).

For simulations of waves propagating in piecewise constant media, DG methods with an interface-fitting triangular mesh can capture material discontinuity more accurately than methods based on unstructured meshes. However, the errors caused by misalignment between straight-sided elements and curved interfaces remain an issue. When triangular meshes are used to fit curved boundaries, any continuous finite-element method for elliptic equations is, at most, second-order accurate for the solution and 1.5-order accurate for the gradient of the solution (Strang and Berger, 1971; Thomée, 1973). DG methods using high-order polynomial bases for hyperbolic equations are at most second-order accurate for the solution (e.g., Bassi and Rebay, 1997). In the presence of curved material interfaces, the same accuracy reduction holds for wave-propagation modeling if we assume homogeneous media on each element (Toulorge et al., 2008; Wang, 2010; Fahs, 2011). In seismic modeling, interface errors may lead to spurious diffractions in the calculated wavefield, particularly when the element size is comparable with the wavelength. The wave energy of the spurious diffractions is much smaller than that of the primary reflections, but is comparable with that of the multiple reflections.

Although the overall error is dominated by the second-order interface error on fine meshes, choosing the order of the polynomial basis depends on the problem (e.g., model, data frequency spectrum, etc.), the available computational resources, and the desired modeling quality. For example, Käser and Dumbser (2006) develop arbitrary high-order polynomial bases and use up to tenth-order polynomial for the Lamb's problem. Etienne et al. (2010) advocate low-order polynomial bases for very complex media. In general, very high-order polynomial bases are popular when a coarse mesh is used because the dispersion error usually causes more harm than the interface error in seismic imaging and inversion. In fact,

Manuscript received by the Editor 9 April 2014; revised manuscript received 19 October 2014; published online 14 January 2015.

${ }^{1}$ Formerly Massachusetts Institute of Technology, Department of Mathematics, Cambridge, Massachusetts, USA; presently Purdue University, Department of Mathematics, West Lafayette, Indiana, USA. E-mail: zhan1966@purdue.edu.

${ }^{2}$ Formerly Los Alamos National Laboratory, Geophysics Group, Los Alamos, New Mexico, USA; presently ExxonMobil Upstream Research Company, Houston, Texas, USA. E-mail: siruitan@hotmail.com.

(C) 2015 Society of Exploration Geophysicists. All rights reserved. 
finite-difference methods with second-order accuracy in time and high-order accuracy in space are widely used, although the interface error is at most first order (Symes and Vdovina, 2009).

To reduce errors caused by curved geometries, curved elements have been developed (e.g., Monk and Wang, 1999; Barucq et al., 2014). For the optimal order of convergence, the standard approach is using curvilinear elements (Gordon and Hall, 1973) to represent curved interfaces. The curvilinear elements are constructed using isoparametric coordinate transforms. Every curvilinear element has its own mass matrix needed to be factored and stored, leading to prohibitive computer-memory requirement when a large number of curvilinear elements are used to fit complex interfaces. Although the computer memory requirement is mitigated by the low-storage curvilinear DG method (Warburton, 2013), significant extra efforts are needed for implementation. We aim to develop a simpler algorithm to reduce the interface errors in this paper.

Krivodonova and Berger (2006) introduce a simple way to approximate curvilinear boundaries and reduce the boundary errors for gas dynamics problems using the correct representation of the normal vectors to the boundary geometry. This approach avoids the use of curvilinear elements. However, we observe from our numerical experiments that extending this approach to interface problems leads to almost the same results as the conventional DG scheme.

We present a simple correction to the DG methods on triangles to reduce errors due to piecewise segment approximation of curved interfaces. We not only use the correct normal vectors to the curved interfaces but also modify the evaluation points for computing numerical fluxes. We demonstrate the effectiveness of our simple correction using numerical modeling of scalar-wave propagation in media with curved interfaces. Our examples demonstrate that our new DG scheme gives errors with much smaller magnitudes compared with the conventional DG scheme, although both the schemes have second-order convergence. Our method is certainly not as accurate as curvilinear elements, but the advantage of our method is its simplicity, especially for problems involving multiple complex interfaces.

\section{DISCONTINUOUS GALERKIN METHODS}

Let $\mathbf{x}=(x, z)$ denote the spatial variables. We consider the following scalar acoustic-wave equation in velocity-stress formulation as

$$
\begin{aligned}
\rho \frac{\partial \mathbf{v}}{\partial t}+\nabla p & =\mathbf{0} \\
\frac{1}{\kappa(\mathbf{x})} \frac{\partial p}{\partial t}+\nabla \cdot \mathbf{v} & =s(\mathbf{x}, t),
\end{aligned}
$$

where $\mathbf{v}=\left(v_{x}, v_{z}\right)$ is the velocity, $p$ is pressure, $\rho$ is the constant density, and $\kappa$ is the bulk modulus. We only consider models composed of piecewise constant media. The wave speed is $c=\sqrt{\kappa / \rho}$. The point source is given by $s(\mathbf{x}, t)=f(t) \delta\left(\mathbf{x}-\mathbf{x}_{s}\right)$, where $\mathbf{x}_{s}$ is the source location and $f(t)$ is the source time function. We can rewrite equation 1 as

$$
\mathbf{w}_{t}+\mathbf{A}(x, z) \mathbf{w}_{x}+\mathbf{B}(x, z) \mathbf{w}_{z}=\left[0,0, \kappa f(t) \delta\left(\mathbf{x}-\mathbf{x}_{s}\right)\right]^{T},
$$

where $\mathbf{w}=\left[v_{x}, v_{z}, p\right]^{T}$ and

$$
\mathbf{A}=\left(\begin{array}{ccc}
0 & 0 & 1 / \rho \\
0 & 0 & 0 \\
\kappa & 0 & 0
\end{array}\right), \quad \mathbf{B}=\left(\begin{array}{ccc}
0 & 0 & 0 \\
0 & 0 & 1 / \rho \\
0 & \kappa & 0
\end{array}\right)
$$

For an interface-fitting triangular mesh, we set $\rho$ and $\kappa$ as constants on each triangle $K$. Multiplying equation 2 by a smooth test function $u$ compactly supported in $K$ and taking an integral over $K$, after integration by parts, we obtain

$$
\int_{K} \mathbf{w}_{t} u \mathrm{~d} V-\int_{K}\left(\frac{\partial u}{\partial x} \mathbf{A}+\frac{\partial u}{\partial z} \mathbf{B}\right) \mathbf{w} \mathrm{d} V+\int_{\partial K} u \mathbf{F} \mathrm{d} s=\mathbf{s}(t) u\left(\mathbf{x}_{s}\right),
$$

where $\mathbf{s}(t)=\left[0,0, \kappa\left(\mathbf{x}_{s}\right) f(t)\right]^{T}$, the flux $\mathbf{F}=\left(n_{x} \mathbf{A}+n_{z} \mathbf{B}\right) \mathbf{w}$, and $\mathbf{n}=\left(n_{x}, n_{z}\right)$ is the outward unit normal vector to the boundary $\partial K$ of the triangle.

In DG methods, we seek piecewise polynomials $\mathbf{w}_{h}$ satisfying the following weak formulation for any piecewise polynomial test function $u_{h}$ :

$$
\begin{aligned}
& \frac{d}{d t} \int_{K} \mathbf{w}_{h} u_{h} \mathrm{~d} V-\int_{K}\left(\frac{\partial u_{h}}{\partial x} \mathbf{A}+\frac{\partial u_{h}}{\partial z} \mathbf{B}\right) \mathbf{w}_{h} \mathrm{~d} V \\
& +\int_{\partial K} u_{h} \hat{\mathbf{F}} \mathrm{d} s=\mathbf{s}(t) u_{h}\left(\mathbf{x}_{s}\right),
\end{aligned}
$$

where the numerical flux $\hat{\mathbf{F}}=\left(n_{x} \mathbf{A}+n_{z} \mathbf{B}\right) \mathbf{w}^{*}$. Here, $\mathbf{w}^{*}$ is an approximation to the values of $\mathbf{w}$ on the boundary because $\mathbf{w}_{h}$ is discontinuous across the boundary $\partial K$. For instance, $\mathbf{w}^{*}$ can be taken as the exact solution of the 1D Riemann problem in the direction normal to each edge of $K$ (Wang, 2010). We denote the three edges of $K$ by $e_{K}^{i}, i=1,2,3$, with outward unit normal vector $\mathbf{n}^{i}$. Let $K_{i}$ denote the neighboring triangle along $e_{K}^{i}$, and $\mathbf{w}_{K}$ denote the polynomial defined on $K$. Then, the numerical flux term $\int_{\partial K} u_{h} \hat{\mathbf{F}} \mathrm{d} s$ can be written as

$$
\begin{aligned}
\int_{\partial K} u_{h} \hat{\mathbf{F}} \mathrm{d} s & =\sum_{i=1}^{3} \int_{e_{K}^{i}} u_{h} \hat{\mathbf{F}} \mathrm{d} s \\
& =\sum_{i=1}^{3} \int_{e_{K}^{i}} u_{h}\left(n_{x}^{i} \mathbf{A}+n_{z}^{i} \mathbf{B}\right) \mathbf{w}^{*}\left[\mathbf{w}^{\operatorname{int}(K)}, \mathbf{w}^{\operatorname{ext}(K)}, \mathbf{n}^{i}\right] \mathrm{d} s,
\end{aligned}
$$

where $\mathbf{w}^{\operatorname{int}(K)}=\mathbf{w}_{K}$ and $\mathbf{w}^{\operatorname{ext}(K)}=\mathbf{w}_{K_{i}}$ for each edge $e_{K}^{i}$ are the approximation values from inside and outside of $K$, respectively. For each edge $e_{K}^{i}$, let $v^{\operatorname{int}(K)}=\mathbf{v}^{\operatorname{int}(K)} \cdot \mathbf{n}^{i}$ and $v^{\operatorname{ext}(K)}=\mathbf{v}^{\operatorname{ext}(K)} \cdot \mathbf{n}^{i}$ denote the normal velocities, then $\mathbf{w}^{*}\left[\mathbf{w}^{\operatorname{int}(K)}, \mathbf{w}^{\operatorname{ext}(K)}, \mathbf{n}^{i}\right]$ denotes the exact solution of the following 1D Riemann problem:

$$
\begin{aligned}
& \rho(\xi) \frac{\partial v}{\partial t}+p_{\xi}=0, \quad \frac{1}{\kappa(\xi)} p_{t}+v_{\xi}=0, \\
& (v(\xi, 0), p(\xi, 0))= \begin{cases}\left(v^{\operatorname{int}(K)}, p^{\operatorname{int}(K)}\right), & \xi<0 \\
\left(v^{\operatorname{ext}(K)}, p^{\operatorname{ext}(K)}\right), & \xi>0\end{cases} \\
& (\rho, \kappa)=\left\{\begin{array}{ll}
\left(\rho_{K}, \kappa_{K}\right), & \xi<0 \\
\left(\rho_{K_{i}}, \kappa_{K_{i}}\right), & \xi>0
\end{array} .\right.
\end{aligned}
$$


Applying integration by parts to equation 5, we obtain the equivalent strong formulation of the DG scheme:

$$
\begin{aligned}
& \int_{K}\left(\frac{\partial \mathbf{w}_{h}}{\partial t}+\mathbf{A} \frac{\partial \mathbf{w}_{h}}{\partial x}+\mathbf{B} \frac{\partial \mathbf{w}_{h}}{\partial z}\right) u_{h} \mathrm{~d} V+\sum_{i=1}^{3} \int_{e_{K}^{i}} u_{h}(\hat{\mathbf{F}}-\mathbf{F}) \mathrm{d} s \\
& \quad=\mathbf{s}(t) u_{h}\left(\mathbf{x}_{s}\right) .
\end{aligned}
$$

The time evolution is solved by the fourth-order low-storage RungeKutta method (Hesthaven and Warburton, 2007).

\section{A SIMPLE AND ACCURATE INTERFACE TREATMENT}

We assume edge $e_{K}^{1}$ of triangle $K$ approximates the curved interface; see Figure 1a for an illustration. We briefly review how to compute flux terms before introducing a simple geometric correction to the flux term $\int_{e_{K}^{1}} u_{h}(\hat{\mathbf{F}}-\mathbf{F}) \mathrm{d} s$ on this edge in equation 8. For convenience, we denote the integrand $u_{h}(\hat{\mathbf{F}}-\mathbf{F})$ as a function of $\mathbf{x} \in e_{K}^{1}$ :

$$
\begin{aligned}
\mathbf{G}\left(\mathbf{x}, \mathbf{n}^{1}\right)= & u_{h}(\mathbf{x})\left(n_{x}^{1} \mathbf{A}_{K}+n_{z}^{1} \mathbf{B}_{K}\right)\left(\mathbf{w}^{*}\left[\mathbf{w}_{K}(\mathbf{x}), \mathbf{w}_{K_{1}}(\mathbf{x}), \mathbf{n}^{1}\right]\right. \\
& \left.-\mathbf{w}_{K}(\mathbf{x})\right) .
\end{aligned}
$$

The line integral is computed by quadratures. We assume that the polynomial degree of $\mathbf{w}_{h}$ is $N$, and we use the $\alpha$-optimized nodal distribution points of triangle $K$ to represent degrees of freedom of all polynomials as discussed by Hesthaven and Warburton (2007). Then, the line integral can be written as

$$
\int_{e_{K}^{1}} u_{h}(\hat{\mathbf{F}}-\mathbf{F}) \mathrm{d} s=\sum_{j=1}^{N+1} \mathbf{G}\left(\mathbf{x}_{j}, \mathbf{n}^{1}\right) \omega_{j},
$$

where $\mathbf{x}_{j}(j=1, \ldots, N+1)$ are the nodal points of $K$ lying on edge $e_{K}^{1}$ and $\omega_{j}$ denotes the corresponding weight with $\sum_{j=1}^{N+1} \omega_{j}$ equal to the length of the edge $e_{K}^{1}$.

We observe the following geometric corrections to equation 10 reduce interface errors:

1) Find the projection points of $\mathbf{x}_{j}$ on the curve, denoted as $\overline{\mathbf{x}}_{j}$. See Figure $1 \mathrm{~b}$ for an illustration.

2) Find a vector normal to the curve at the projection points $\overline{\mathbf{x}}_{j}$. For instance, we can use $\overline{\mathbf{x}}_{j}-\mathbf{x}_{j}$. The outward unit normal vector is denoted by $\mathbf{n}\left(\overline{\mathbf{x}}_{j}\right)$.

3) Replace $\mathbf{G}\left(\mathbf{x}_{j}, \mathbf{n}^{1}\right)$ by $\mathbf{G}\left(\overline{\mathbf{x}}_{j}, \mathbf{n}\left(\overline{\mathbf{x}}_{j}\right)\right)$ in equation 10; i.e.,

$$
\int_{e_{K}^{1}} u_{h}(\hat{\mathbf{F}}-\mathbf{F}) d s=\sum_{j=1}^{N+1} \mathbf{G}\left(\overline{\mathbf{x}}_{j}, \mathbf{n}\left(\overline{\mathbf{x}}_{j}\right)\right) \omega_{j}
$$

Let $\Gamma$ denote the curve in Figure 1a. In the last step of this correction, notice that $\sum_{j=1}^{N+1} \mathbf{G}\left(\overline{\mathbf{x}}_{j}, \mathbf{n}\left(\overline{\mathbf{x}}_{j}\right)\right) \omega_{j}$ in equation 11 is not a high-order accurate approximation of the integral
$\int_{\Gamma} u_{h}(\hat{\mathbf{F}}-\mathbf{F}) \mathrm{d} s=\int_{\Gamma} \mathbf{G}(\mathbf{x}, \mathbf{n}) \mathrm{d} s$ because the arc length of the curve $\Gamma$ is not included. Intuitively, the correction would be more accurate if we replaced $\int_{e_{K}^{1}} u_{h}(\hat{\mathbf{F}}-\mathbf{F}) \mathrm{d} s$ in equation 11 with an accurate approximation of $\int_{\Gamma} u_{h}(\hat{\mathbf{F}}-\mathbf{F}) \mathrm{d} s$. However, the function $(\hat{\mathbf{F}}-\mathbf{F})$ becomes zero on the interface curve $\Gamma$ if $\mathbf{w}_{K}$ and $\mathbf{w}_{K_{1}}$ are equal to the exact solution in equation 9 . Thus, $\int_{\Gamma} u_{h}(\hat{\mathbf{F}}-\mathbf{F}) d s$ can be regarded as a penalty term approximating zero. When replacing the numerical solution $\mathbf{w}_{K}$ and $\mathbf{w}_{K_{1}}$ with the exact solution in equation $9, \sum_{j=1}^{N+1} \mathbf{G}\left(\overline{\mathbf{x}}_{j}, \mathbf{n}\left(\overline{\mathbf{x}}_{j}\right)\right) \omega_{j}$ in equation 11 is also zero, but $\int_{e_{K}^{1}} u_{h}(\hat{\mathbf{F}}-\mathbf{F}) \mathrm{d} s$ is second order away from zero. Thus, $\sum_{j=1}^{N+1} \mathbf{G}\left(\overline{\mathbf{x}}_{j}, \mathbf{n}\left(\overline{\mathbf{x}}_{j}\right)\right) \omega_{j}$ is a good approximation to the penalty term $\int_{\Gamma} u_{h}(\hat{\mathbf{F}}-\mathbf{F}) \mathrm{d} s$ from the point of view of the local truncation error, which explains why this correction may reduce interface errors. In our numerical tests, we did not observe any meaningful improvement by including the arc length in equation 11 . Therefore, we recommend using the simple correction without involving the arc length.

We apply this correction for all edges approximating the curve. Our correction strategy is different from the one in Krivodonova and Berger (2006). Their method is developed for the solid wall boundary condition in gas dynamics problems. To extend the method to interface problems, one may replace the normal vector in the flux term $\int_{e_{K}^{i}} u_{h} \hat{\mathbf{F}} \mathrm{d} s$ in equation 6 with the correct normal vector to the interface geometry. However, our numerical tests suggest that such correction may not be consistent with the wave equation. A consistent correction is using the correct normal vector in the term $\int_{e_{K}^{i}} u_{h}(\hat{\mathbf{F}}-\mathbf{F}) \mathrm{d} s$ in equation 8. Our numerical tests show that this correction hardly changes the modeling results obtained using the conventional DG scheme.

For highly curved interfaces, we have to locally refine the mesh such that the mesh size is sufficiently smaller than the radius of curvature. When the mesh size approaches zero, we obtain the asymptotic convergence rate of second order. For a given radius of curvature, it is very difficult to quantitatively analyze the maximum allowed mesh size for numerical stability and accuracy. Such analyses are out of the scope of this paper.

\section{NUMERICAL EXAMPLES}

We compare the accuracy of our simple geometric correction with that of the conventional DG scheme. We do not show the re- a)

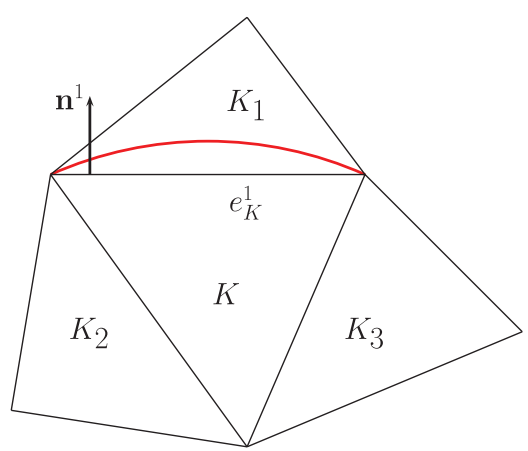

b)

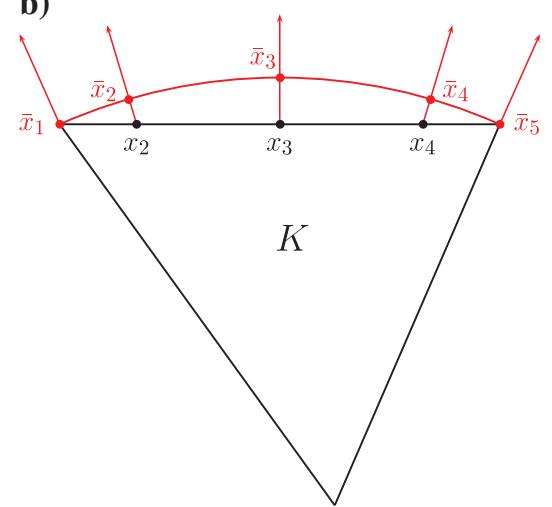

Figure 1. An illustration of (a) an element near the curved interface and (b) the projection of the quadrature points to the interface. The red curve is the material interface. 
sults obtained using the method of Krivodonova and Berger (2006) because they are almost the same as those of the conventional DG scheme in all our numerical examples. In the first set of examples, the maximum errors of both methods are exactly the same within three significant figures. In the second set of examples, there is no visible difference between the common-shot gathers obtained using both methods.

\section{Media with circular interfaces}

We consider scattering of a plane wave by a cylinder with the given analytical solution to test the accuracy of the geometric correction. The cylinder is assumed to have a radius of $r_{0}=0.6$. Outside the cylinder, i.e., $\sqrt{x^{2}+z^{2}}=r<r_{0}$, the density is constant $\rho_{1}$ and the bulk modulus is constant $\kappa_{1}$. For $r \geq r_{0}$, the density and bulk modulus are constants $\rho_{2}$ and $\kappa_{2}$, respectively. The source term $s(\mathbf{x}, t)=0$ in equation 1 . Assume the cylinder is illuminated by a plane wave of the following form:

$p^{\mathrm{inc}}=\exp \left(-\mathrm{i}\left(k_{1} x-\omega t\right)\right), \quad u^{\mathrm{inc}}=\exp \left(-\mathrm{i}\left(k_{1} x-\omega t\right)\right)$,

$v^{\text {inc }}=0$,

where $k_{1}=\omega \sqrt{\rho_{1} / \kappa_{1}}$. Then, the problem has an exact solution given as follows (Cai and Deng, 2003):
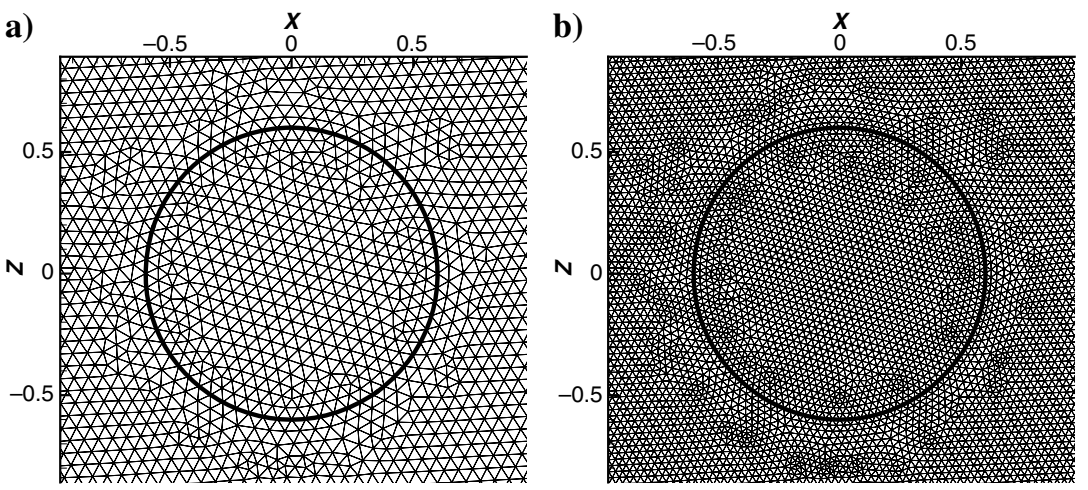

Figure 2. (a) Mesh 1 and (b) mesh 2 for DG modeling. Both meshes fit the circular material interface indicated by heavy curves.

$$
\begin{aligned}
& p(x, z, t)=p(r, \theta, t) \\
& =e^{\mathrm{i} \omega t}\left\{\begin{array}{cc}
\sum_{n=-\infty}^{\infty} C_{n}^{t o t} J_{n}\left(k_{2} r\right) e^{\mathrm{i} n \theta}, & r \leq r_{0}, \\
\sum_{n=-\infty}^{\infty}\left(\mathrm{i}^{-n} J_{n}\left(k_{1} r\right)+C_{n}^{\mathrm{scat}} H_{n}\left(k_{1} r\right)\right) e^{\mathrm{i} n \theta}, & r>r_{0}
\end{array},\right.
\end{aligned}
$$

where $(r, \theta)=\left(\sqrt{x^{2}+z^{2}}, \arctan (z / x)\right)$ represent the polar coordinates, $J_{n}$ and $H_{n}$ represent the $n$ th-order Bessel function of the first kind and the Hankel function of the second kind, respectively, and $k_{2}=\omega \sqrt{\rho_{2} / \kappa_{2}}$. The expansion coefficients are given as

$$
C_{n}^{\mathrm{tot}}=\mathrm{i}^{-n} \frac{\frac{k_{1}}{\rho_{1}} J_{n}{ }^{\prime}\left(k_{1} r_{0}\right) H_{n}\left(k_{1} r_{0}\right)-\frac{k_{1}}{\rho_{1}} H_{n}{ }^{\prime}\left(k_{1} r_{0}\right) J_{n}\left(k_{1} r_{0}\right)}{\frac{k_{2}}{\rho_{2}} J_{n}{ }^{\prime}\left(k_{2} r_{0}\right) H_{n}\left(k_{1} r_{0}\right)-\frac{k_{1}}{\rho_{1}} H_{n}{ }^{\prime}\left(k_{1} r_{0}\right) J_{n}\left(k_{2} r_{0}\right)}
$$

and

$$
C_{n}^{\text {scat }}=\mathrm{i}^{-n} \frac{\frac{k_{1}}{\rho_{1}} J_{n}{ }^{\prime}\left(k_{1} r_{0}\right) J_{n}\left(k_{2} r_{0}\right)-\frac{k_{2}}{\rho_{2}} J_{n}{ }^{\prime}\left(k_{2} r_{0}\right) J_{n}\left(k_{1} r_{0}\right)}{\frac{k_{2}}{\rho_{2}} J_{n}{ }^{\prime}\left(k_{2} r_{0}\right) H_{n}\left(k_{1} r_{0}\right)-\frac{k_{1}}{\rho_{1}} H_{n}{ }^{\prime}\left(k_{1} r_{0}\right) J_{n}\left(k_{2} r_{0}\right)} .
$$

Plug the pressure into equation 1 , then we can obtain the expressions for the velocity.

The computational domain is $[-1.5,1.5] \times[-1.5,1.5]$, and we use perfectly matched layers (PMLs) of width 0.25 to terminate the computation at the boundaries. We set $\omega=2 \pi$ and use the real part of the analytical solution to test the accuracy of DG schemes. The final time is set as $T=0.4$, and we monitor the maximum error over the nodal points of each triangle inside the square $[-0.8,0.8] \times[-0.8,0.8]$ to exclude possible errors due to the PML. Mesh 1 and mesh 2 used in the numerical tests are shown in Figure 2. Mesh 3 is a straightforward refinement of mesh 2 . We consider two cases with different velocity contrasts. One is with $\rho_{1}=\kappa_{1}=$ $\rho_{2}=1$ and $\kappa_{2}=1 / 2.25$ such that the velocity contrast is $1: 1.5$. We take $\rho_{1}=\kappa_{1}=\rho_{2}=1$ and $\kappa_{2}=1 / 25$ for the other case such that the velocity contrast is $1: 5$.

The errors for the low-contrast case are listed in Table 1, and the errors for the high-contrast

Table 1. Errors for DG schemes with polynomials of degree $N$. The interface geometry is a circle, and the velocity contrast is 1:1.5. Our new DG scheme gives errors with much smaller magnitudes compared with the conventional DG scheme although both schemes have second-order convergence.

Conventional DG

Modified DG

\begin{tabular}{ccccccccccc}
\hline $\mathrm{N}$ & Mesh 1 & Mesh 2 & Order & Mesh 3 & Order & Mesh 1 & Mesh 2 & Order & Mesh 3 & Order \\
\hline 2 & $7.13 \mathrm{E}-3$ & $1.41 \mathrm{E}-3$ & 2.33 & $3.03 \mathrm{E}-4$ & 2.22 & $3.57 \mathrm{E}-3$ & $4.78 \mathrm{E}-4$ & 2.89 & $6.64 \mathrm{E}-5$ & 2.85 \\
3 & $4.11 \mathrm{E}-3$ & $1.03 \mathrm{E}-3$ & 1.99 & $2.58 \mathrm{E}-4$ & 2.00 & $4.57 \mathrm{E}-4$ & $9.94 \mathrm{E}-5$ & 2.20 & $2.27 \mathrm{E}-5$ & 2.13 \\
4 & $4.11 \mathrm{E}-3$ & $1.03 \mathrm{E}-3$ & 2.00 & $2.58 \mathrm{E}-4$ & 2.00 & $3.30 \mathrm{E}-4$ & $8.36 \mathrm{E}-5$ & 1.98 & $1.94 \mathrm{E}-5$ & 2.11 \\
5 & $4.12 \mathrm{E}-3$ & $1.03 \mathrm{E}-3$ & 2.00 & $2.58 \mathrm{E}-4$ & 2.00 & $2.59 \mathrm{E}-4$ & $7.02 \mathrm{E}-5$ & 1.88 & $1.75 \mathrm{E}-5$ & 2.00 \\
6 & $4.12 \mathrm{E}-3$ & $1.03 \mathrm{E}-3$ & 2.00 & $2.58 \mathrm{E}-4$ & 2.00 & $2.34 \mathrm{E}-4$ & $6.38 \mathrm{E}-5$ & 1.88 & $1.59 \mathrm{E}-5$ & 2.00 \\
7 & $4.13 \mathrm{E}-3$ & $1.03 \mathrm{E}-3$ & 2.00 & $2.58 \mathrm{E}-4$ & 2.00 & $2.01 \mathrm{E}-4$ & $5.66 \mathrm{E}-5$ & 1.83 & $1.43 \mathrm{E}-5$ & 1.98 \\
\hline
\end{tabular}


case are listed in Table 2. In Table 1, the error of the conventional DG scheme decreases significantly when the order of the polynomial basis is increased from $N=2$ to $N=3$ on mesh 1 . When the order of the polynomial basis is further increased, the overall errors are almost unchanged on mesh 1 . This is because the interface error dominates, and the conventional high-order DG scheme is not able to reduce the interface error without grid refinement. We can see that the simple geometric correction reduces the error magnitude in Tables 1 and 2. Even though there is an apparent reduction in the order of convergence for mesh 2 in the low-contrast case, the convergence rate for the further refined mesh 3 is approximately second order for both the DG schemes with polynomials of degree $N \geq 2$.

For comparison, we list the errors of the curvilinear element method in Table 3. In general, the curvilinear element method gives smaller errors and faster convergence rates than our modified DG method. However, in some cases, the errors of the two methods are similar. For example, in the case of velocity contrast $1: 1.5$, the error of our method with the polynomial of degree $N=2$ is $3.57 \mathrm{E}-3$ on mesh 1 , whereas the error of the curvilinear element method with the same $N$ is $3.37 \mathrm{E}-3$ on mesh 1 . In the case of velocity contrast $1: 5$, the errors of our method with polynomials of degrees $N=2$

Table 2. Errors for DG schemes with polynomials of degree $N$. The interface geometry is a circle, and the velocity contrast is 1:5. Our new DG scheme gives much smaller errors than the conventional DG scheme.

Conventional DG

Modified DG

\begin{tabular}{ccccccccccc}
\hline $\mathrm{N}$ & Mesh 1 & Mesh 2 & Order & Mesh 3 & Order & Mesh 1 & Mesh 2 & Order & Mesh 3 & Order \\
\hline 2 & $1.12 \mathrm{E}-1$ & $1.42 \mathrm{E}-2$ & 2.98 & $2.39 \mathrm{E}-3$ & 2.57 & $1.01 \mathrm{E}-1$ & $1.18 \mathrm{E}-2$ & 3.09 & $1.65 \mathrm{E}-3$ & 2.84 \\
3 & $2.38 \mathrm{E}-2$ & $4.03 \mathrm{E}-3$ & 2.56 & $9.98 \mathrm{E}-4$ & 2.01 & $1.51 \mathrm{E}-2$ & $1.05 \mathrm{E}-3$ & 3.84 & $2.48 \mathrm{E}-4$ & 2.08 \\
4 & $1.62 \mathrm{E}-2$ & $3.98 \mathrm{E}-3$ & 2.02 & $9.95 \mathrm{E}-4$ & 2.00 & $3.88 \mathrm{E}-3$ & $8.81 \mathrm{E}-4$ & 2.14 & $2.24 \mathrm{E}-4$ & 1.97 \\
5 & $1.62 \mathrm{E}-2$ & $3.98 \mathrm{E}-3$ & 2.02 & $9.96 \mathrm{E}-4$ & 2.00 & $3.24 \mathrm{E}-3$ & $8.03 \mathrm{E}-4$ & 2.01 & $1.95 \mathrm{E}-4$ & 2.04 \\
6 & $1.62 \mathrm{E}-2$ & $3.98 \mathrm{E}-3$ & 2.02 & $9.96 \mathrm{E}-4$ & 2.00 & $2.70 \mathrm{E}-3$ & $6.93 \mathrm{E}-4$ & 1.96 & $1.83 \mathrm{E}-4$ & 1.92 \\
7 & $1.62 \mathrm{E}-2$ & $3.98 \mathrm{E}-3$ & 2.03 & $9.96 \mathrm{E}-4$ & 2.00 & $2.40 \mathrm{E}-3$ & $6.23 \mathrm{E}-4$ & 1.95 & $1.67 \mathrm{E}-4$ & 1.90 \\
\hline
\end{tabular}

Table 3. Errors for DG schemes using curvilinear elements with polynomials of degree $N$. The interface geometry is a circle. In general, the curvilinear element method gives smaller errors and faster convergence rates than our modified DG method. However, in some cases, the errors of the two methods are similar.

Velocity contrast $1: 1.5$

Velocity contrast $1: 5$

\begin{tabular}{ccccccccccc}
\hline $\mathrm{N}$ & Mesh 1 & Mesh 2 & Order & Mesh 3 & Order & Mesh 1 & Mesh 2 & Order & Mesh 3 & Order \\
\hline 2 & $3.37 \mathrm{E}-3$ & $4.72 \mathrm{E}-4$ & 2.99 & $5.96 \mathrm{E}-5$ & 2.99 & $9.99 \mathrm{E}-2$ & $1.18 \mathrm{E}-2$ & 3.07 & $1.65 \mathrm{E}-3$ & 2.84 \\
3 & $1.88 \mathrm{E}-4$ & $1.37 \mathrm{E}-5$ & 3.78 & $8.88 \mathrm{E}-7$ & 3.95 & $1.51 \mathrm{E}-2$ & $1.00 \mathrm{E}-3$ & 3.91 & $6.84 \mathrm{E}-5$ & 3.87 \\
4 & $7.02 \mathrm{E}-6$ & $2.24 \mathrm{E}-7$ & 4.97 & $7.03 \mathrm{E}-9$ & 4.99 & $1.95 \mathrm{E}-3$ & $5.92 \mathrm{E}-5$ & 5.04 & $2.08 \mathrm{E}-6$ & 4.83 \\
5 & $1.68 \mathrm{E}-7$ & $3.41 \mathrm{E}-9$ & 5.62 & $5.60 \mathrm{E}-11$ & 5.93 & $1.61 \mathrm{E}-4$ & $2.99 \mathrm{E}-6$ & 5.75 & $5.09 \mathrm{E}-8$ & 5.88 \\
6 & $1.54 \mathrm{E}-7$ & $3.70 \mathrm{E}-11$ & 12.01 & $8.95 \mathrm{E}-13$ & 5.37 & $1.62 \mathrm{E}-5$ & $1.30 \mathrm{E}-7$ & 6.96 & $1.13 \mathrm{E}-9$ & 6.85 \\
7 & $1.22 \mathrm{E}-7$ & $1.44 \mathrm{E}-11$ & 13.04 & $6.84 \mathrm{E}-14$ & 7.72 & $9.54 \mathrm{E}-7$ & $4.26 \mathrm{E}-9$ & 7.80 & $1.83 \mathrm{E}-11$ & 7.86 \\
\hline
\end{tabular}

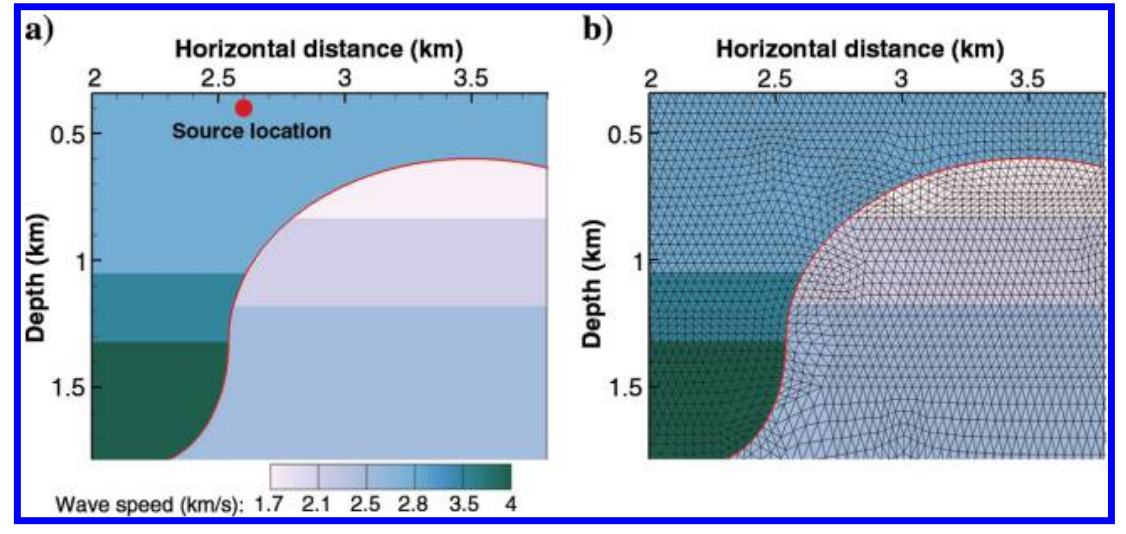

Figure 3. (a) A 2D dome model and (b) the interface-fitting triangular mesh used for modeling. 

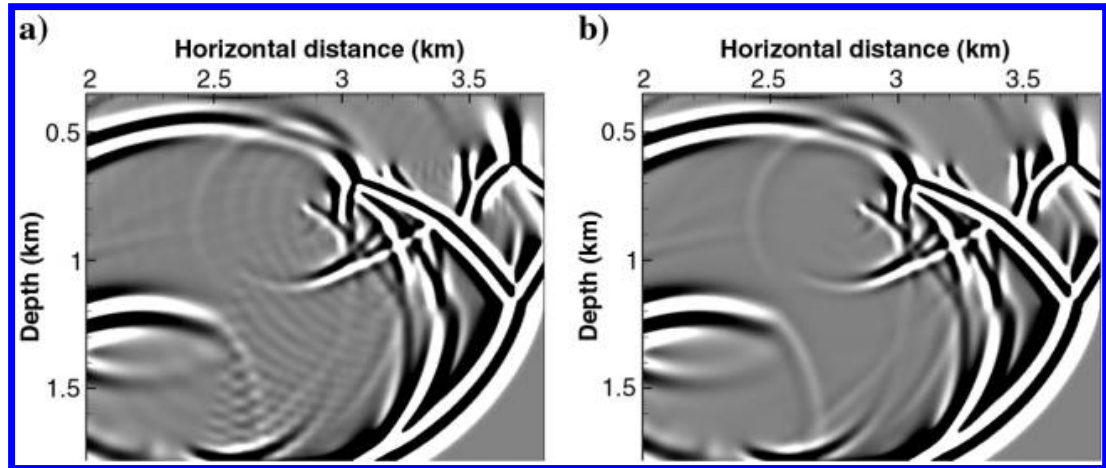

Figure 4. Snapshots of the pressure wavefield at $T=0.7 \mathrm{~s}$ obtained using (a) the conventional DG scheme and (b) our DG scheme with simple geometric correction. The snapshot in panel (a) contains spurious diffractions caused by interface error. Our geometric correction significantly suppresses the spurious diffractions in panel (b).

a)

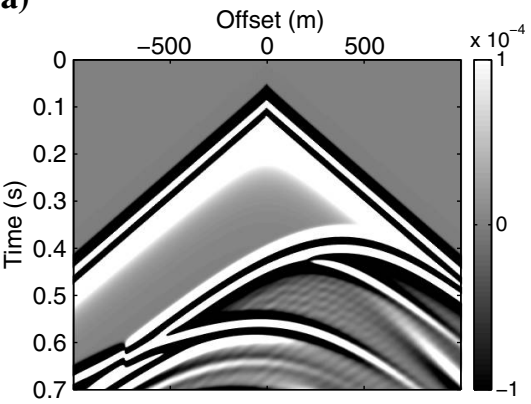

b)

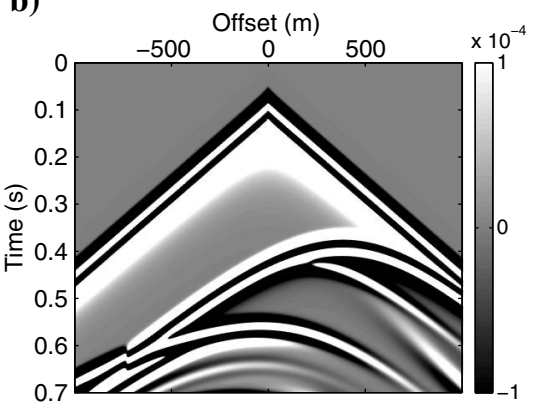

Figure 5. Common-shot gather obtained using (a) the conventional DG scheme and (b) our DG scheme with simple geometric correction. The spurious diffractions in panel (a) are almost invisible in panel (b). and $N=3$ are almost the same as those of the curvilinear element method with the same mesh. Thus, for some problems and certain given error thresholds, the performance of our DG method is similar to that of the curvilinear element method.

\section{Dome model}

We consider a 2D dome model illustrated in Figure $3 \mathrm{a}$ in which the density is constant everywhere. The model is similar to the one used by Wang (2010). A Ricker-wavelet source with a central frequency of $25 \mathrm{~Hz}$ is located at $(3300 \mathrm{~m}, 40 \mathrm{~m})$. All 400 receivers lie uniformly on the line from $(2300 \mathrm{~m}, 40 \mathrm{~m})$ to $(4300 \mathrm{~m}$, $40 \mathrm{~m}$ ).

We test DG methods with a polynomial of degree 8 on the mesh shown in Figure $3 b$. This high polynomial order is necessary to ensure that there is no visible dispersion error in the modeled wavefields. Along the curved interface, the length of the edges of the triangles ranges from 35 to $42 \mathrm{~m}$, which is around the minimum wavelength. The conventional DG scheme on triangles produces spurious diffractions on this relatively coarse mesh due to the piecewise segment approximation to the curve. In Figure 4a, we observe nonphysical scattering generated near the curved interface. We then apply the simple geometric correction to the conventional DG scheme. The numerical diffractions are significantly suppressed (Figure $4 \mathrm{~b}$ ). The spurious diffractions generated by the conventional DG scheme also appear in the common-shot gather shown in Figure 5a. The spurious diffractions are almost invisible in the common-shot gather obtained using our geometric correction (Figure 5b). Figure 6 displays traces obtained using both schemes at the receiver with an offset of $250 \mathrm{~m}$. The reference trace is obtained using the conventional DG scheme with a polynomial of degree 9 on a refined mesh. The trace obtained using our geometric correction matches well with the reference one, whereas the conventional DG scheme gives spurious oscillations. The computational cost of our scheme is much smaller than that of the conventional DG scheme on the refined mesh.

\section{CONCLUSIONS}

We developed a novel DG scheme for modeling scalar-wave propagation in media with curved interfaces. As the conventional DG scheme, our scheme is based on straight-sided triangular elements. To account for curved interfaces, we use the correct normal vectors and modify the numerical fluxes. Numerical examples demonstrate that our new DG scheme gives much smaller errors compared with the conventional scheme, although both schemes have second-order convergence. For the dome model, the conventional scheme generates spurious diffractions caused by interface errors, whereas our scheme significantly suppresses the spurious diffractions. Our scheme gives a wavefield similar to that obtained using the conventional scheme on a refined mesh, but with a much smaller computational cost than the latter. Our new DG scheme is, thus,
Figure 6. Traces obtained using the conventional DG scheme (plus symbols) and our DG scheme (circle symbols) at the receiver with an oset of $250 \mathrm{~m}$. The solid curve shows the reference trace. The trace obtained using our DG scheme matches well with the reference one, whereas the conventional DG scheme gives spurious oscillations. 
particularly useful for large-scale scalar-wave modeling involving complex subsurface structures.

\section{ACKNOWLEDGMENTS}

The work of S. Tan was supported by the U.S. Department of Energy through contract no. DE-AC52-06NA25396 to Los Alamos National Laboratory. We thank the associate editor and two anonymous reviewers for their valuable comments.

\section{REFERENCES}

Barucq, H., R. Djellouli, and E. Estecahandy, 2014, Efficient DG-like formulation equipped with curved boundary edges for solving elasto-acoustic scattering problems: International Journal for Numerical Methods in Engineering, 98, 747-780, doi: 10.1002/nme.4652.

Bassi, F., and S. Rebay, 1997, High-order accurate discontinuous finite element solution of the 2D Euler equations: Journal of Computational Physics, 138, 251-285, doi: 10.1006/jcph.1997.5454.

Cai, W., and S. Deng, 2003, An upwinding embedded boundary method for Maxwell's equations in media with material interfaces: 2D case: Journal of Computational Physics, 190, 159-183, doi: 10.1016/S0021-9991(03) 00269-9.

Cockburn, B., and C.-W. Shu, 1989, TVB Runge-Kutta local projection discontinuous Galerkin finite element method for conservation laws Part II: General framework: Mathematics of Computation, 52, 411-435.

Etienne, V., E. Chaljub, J. Virieux, and N. Glinsky, 2010, An hp-adaptive discontinuous Galerkin finite-element method for 3D elastic wave modelling: Geophysical Journal International, 183, 941-962, doi: 10.1111/j $.1365-246 X .2010 .04764 . x$.

Fahs, H., 2011, Improving accuracy of high-order discontinuous Galerkin method for time-domain electromagnetics on curvilinear domains: International Journal of Computer Mathematics, 88, 2124-2153, doi: 10.1080/00207160.2010.527960.
Gordon, W., and C. Hall, 1973, Transfinite element methods: Blendingfunction interpolation over arbitrary curved element domains: Numerische Mathematik, 21, 109-129, doi: 10.1007/BF01436298.

Hesthaven, J., and T. Warburton, 2007, Nodal discontinuous Galerkin methods: Algorithms, analysis, and applications: Springer.

Käser, M.,, and M. Dumbser, 2006, An arbitrary high-order discontinuous Galerkin method for elastic waves on unstructured meshes - Part I: The two-dimensional isotropic case with external source terms: Geophysical Journal International, 166, 855-877, doi: 10.1111/j.1365-246X.2006 $.03051 . x$.

Krivodonova, L., and M. Berger, 2006, High-order accurate implementation of solid wall boundary conditions in curved geometries: Journal of Computational Physics, 211, 492-512, doi: 10.1016/j.jcp.2005.05.029.

Monk, P., and D.-Q. Wang, 1999, A least-squares method for the Helmholtz equation: Computer Methods in Applied Mechanics and Engineering, 175, 121-136, doi: 10.1016/S0045-7825(98)00326-0.

Reed, W., and T. Hill, 1973, Triangular mesh methods for the neutron transport equation: Los Alamos Scientific Laboratory, report LA-UR-73-479.

Strang, G., and A. E. Berger, 1971, The change in solution due to change in domain, in D. C. Spencer, ed., AMS Symposium on Partial Differential Equations: American Mathematical Society, 199-206.

Symes, W., and T. Vdovina, 2009, Interface error analysis for numerical wave propagation: Computational Geosciences, 13, 363-371, doi: 10 1007/s10596-008-9124-8.

Thomée, V., 1973, Polygonal domain approximation in Dirichlet's problem: IMA Journal of Applied Mathematics, 11, 33-44, doi: 10.1093/imamat/ 11.1.33.

Toulorge, T., Y. Reymen, and W. Desmet, 2008, A 2D discontinuous Galerkin method for aeroacoustics with curved boundary treatment, in P. Sas, and B. Bergen, eds., Proceedings of the International Conference on Noise and Vibration Engineering, Katholieke Universiteit Leuven, 565-578.

Wang, X., 2010, Discontinuous Galerkin time domain methods for acoustics and comparison with finite difference time domain methods: M.S. thesis, Rice University.

Warburton, T., 2013, A low-storage curvilinear discontinuous Galerkin method for wave problems: SIAM Journal on Scientific Computing, 35, A1987-A2012, doi: 10.1137/120899662. 